\title{
LA HEMORRAGIA POST-PARTUM(*)
}

\section{Doctor J. Afanador Ruiz}

La hemorragia uterina post-partum es una de las mayores complicaciones en obstetricia y la principal causa de muerte materna. Sus causas han sido cuidadosamente estudiadas en los últi_ mos años, y la más común es la atonía uterina, es decir, la defi_ ciente contracción del útero inmediatamente después del parto la cual puede ser el resultado de una retención placentaria, un fibro_ ma intramural, sobredistensión uterina, anestesia profunda, esta_ dos de debilidad general y shock.

No pocos son los casos de hemorragias post_partum debidas a lesiones traumáticas del canal genital.

Existen otros casos de hemorragias uterinas fatales en los cuales se presenta un desorden de la coagulación y últimamente se han descrito alteraciones de los mecanismos hemostáticos en varios estados patológicos.

Las estadisticas americanas de 1950 revelan 1.044 muertes atribuíbles a hemorragias durante el embarazo o inmediatamente después del parto; además se estima que el $50 \%$ de las mujeres que mueren por infección puerperal han tenido hemorragias ex. cesivas que no han sido tratadas.

Se acepta la existencia de hemorragia post-partum cuando la cantidad de sangre perdida después de la expulsión de la placenta es superior a 500 c. c.

Normalmente el flujo sanguíneo a la placenta se sostiene de. bido a la distensión uterina producida por el feto, pero una vez que el niño nace y se expulsa la placenta, las fibras musculares uterinas se contraen firmemente, comprimen los vasos produciéndo_ se así un mecanismo hemostático y posteriormente la sangre se coagula; los vasos sanguíneos se retraen y la hemorragia es míni_

(*) Conferencia de actualización dictada por el autor en el curso para post_graduados organizado por la Universidad de América (1957). 
ma, a pesar de que la fibra uterina se relaje, de esta manera la cantidad de sangre perdida después de un parto normal está en función de estos mecanismos.

El papel que tienen los procesos hemostáticos en el con. trol de la hemorragia del alumbramiento no está bien claro y fue negado hasta hace poco, pero en la actualidad gracias al estudio de Greenberg se ha demostrado que la sangre que sale inmediata. mente después de la expulsión de la placenta es incoagulable y Barnes posteriormente comprobó que esta sangre no se coagula a pesar de agregársele Tromboplastina, lo cual es indicativo de que carece de fibrinógeno, pero si además se agrega esta sustancia tampoco se produce el coágulo y únicamente se logra mediante la adición de Fibrinógeno y Tromboplastina; por otra parte el coágu. 10 así formado no se destruye, es decir, no entra en lisis. La ob. servación demuestra también que la administración de Dicumarol y Heparina en dosis terapéuticas no aumenta la hemorragia en el curso del puerperio; los datos obtenidos por Greenberg y Barnes pueden explicarse por la formación de coágulos dentro de los va. sos uterinos en el sitio de inserción de la placenta, es decir, que el Fibrinógeno y la Tromboplastina se consumirian en este sitio y la sangre del alumbramiento es incoagulable por este mecanismo.

\section{CLASIFICACION}

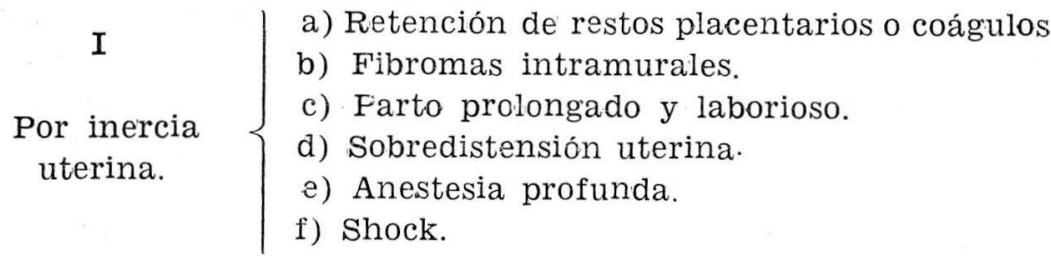

II

Traumática.
$\left\{\begin{array}{l}\text { a) Vulva. } \\ \text { b) Vagina. }\end{array}\right.$
c) Cuello, segmento y cuerpo uterino.
III
a) Por embolía amniótica.
Alteraciones de
b) Desprendimiento de la placenta normal_ mente insertada.
la coagulación
c) Retención prolongada de feto muerto. 
Prevención de la hemorragia "post-partum".-Una excesiva pérdida sanguínea puede en algunos casos ser prevenida si el mé. dico reconoce previamente algunas condiciones durante el emba. razo y lo mismo si durante el parto se logran corregir las condicio. nes capaces de asociarse a estados hemorrágicos.

Cuidados prenatales.-Debe sospecharse la posibilidad de una hemorragia abundante durante el parto en aquellas enfermas con antecedentes hemorrágicos en partos anteriores; 10 mismo que en aquellas que presentan un útero sobredistendido, bien sea por hidramnios o por embarazos múltiples; igual cosa sucede en aquellos casos en los cuales existe una desproporción cefa-pélvica $y$ en los que se prevee un parto laborioso. Durante el embarazo se debe controlar y tratar todo estado anémico, lo mismo que toda de. ficiencia alimenticia.

Durante el parto se debe tratar de corregir todas aquellas anomalías que puedan conducir a un parto prolongado y así se procederá a la ruptura de las membranas o al estímulo de la con. tracción uterina mediante pitocín en dextrosa al 5\% por vía en. dovenosa cuando exista una inercia uterina. Los procedimientos operatorios deberán ser los estrictamente indispensables y los más inocuos. En los casos en que es imposible evitar el parto prolonga. co es muy importante combatir la deshidratación de la paciente, ya que en la hemoconcentración las pérdidas sanguíneas tienen consecuencias más graves. La deshidratación debe tratarse me. diante dextrosa en solución al $5 \%$ por vía endovenosa; los líqui_ dos por vía oral deben limitarse, lo mismo que cualquier alimen. to sólido, pues es sabido que una vez iniciado el trabajo de parto se suspende la asimilación por la mucosa intestinal. El uso de se. dantes y analgésicos debe ser moderado, pues su abuso puede con_ ducir a la necesidad de emplear procedimientos operatorios, los cuales aumentan la posibilidad de hemorragia.

Es conveniente limitar los procedimientos operatorios, y si éstos se imponen deben ser los estrictamente indispensables, reu_ niendo todos los requisitos necesarios, especialmente en cuanto a la aplicación de fórceps se refiere. En los casos en los cuales ei parto se termine con fórceps, la anestesia debe ser lo suficiente. mente profunda para permitir una buena relajación muscular, evi. tando así lesiones de los tejidos blandos. La manera como se con_ duzca el período de desprendimento es también muy importante para prevenir hemorragias durante el alumbramiento. 
A medida que al feto desciende por la vagina va abandonando ia cavidad uterina de tal manera que el útero tiene que hacerse cada vez más pequeño para poder sostener una fuerza expulsiva adecuada, para llenar este cometido la fibra uterina se retrae y se hace cada vez más corta a medida que la presentación avanza hacia la vagina; la retracción uterina se hace gradualmente, pe. ro si por el contrario el útero se vacía súbitamente, el músculo no puede contraerse eficazmente sino hasta que la fibra haya dis. minuído en longitud; de esta manera si el feto nace lentamente con varias pausas durante' su extracción, la fibra muscular uteri_ na tendrá oportunidad de acortarse y de contraerse más eficaz. mente, reduciendo así la hemorragia.

Una vez que la cabeza ha nacido se debe permitir una pausa de unos 30 a 60 segundos para después hacer la extracción de los hombros y luego el resto del feto lentamente. Las contracciones uterinas que se presentan después de la expulsión del niño produ. cen el desprendimiento y expulsión de la placenta, mientras ésta se encuentra en el segmento superior, el diámetro transverso ute rino es mayor que el antero_posterior, pero una vez que se inicia el desprendimiento, el útero se hace globular; cuando la placenta se desprende y cae al segmento inferior, la matriz se hace redon_ da: firme y mediante el pujo se expulsa. Algunos autores aconse. jan ayudar mediante suaves masajes uterinos al mecanismo de desprendimiento placentario; estos masajes se deben hacer du. rante la contracción uterina, tratando de hacer presión hacia aba. jo con mucha suavidad. Cuando la placenta ha salido debe inspec. cionarse para constatar su integridad y si se nota la ausencia de un cotiledón se debe proceder a extraerlo inmediatamente. Es de rutina el empleo de los occitócicos de tipo Ergotrate o Methergin por vía intravenosa para estimular y sostener la contracción ute. rina. El momento de la aplicación de esta droga debe ser induda_ blemente después de la expulsión de la placenta, puesto que isi se aplica antes y se necesita posteriormente hacer una revisión ute_ rina o una extracción de restos placentarios, la exploración de la cavidad se hace extremadamente difícil, necesitando una aneste. sia profunda, lo cual puede ser a su vez factor de hemorragia.

Los estudios sobre la contracción uterina durante el alumbra. miento, hechos por el uruguayo Alvarez, han establecido que el llamado reposo fisiológico del útero después del nacimiento del fe. to no existe, y que la fibra uterina sigue contrayéndose sin inte. rrupción 
El desprendimiento de la placenta debe dejarse exclusivamen_ te a cargo de la contracción uterina; la llamada maniobra de Credé debe abandonarse de la práctica obstétrica, ya que produ. ce shock por reflejo vagal. Cuando la placenta no se desprende después de un tiempo prudencial, deberá hacerse la extracción manual y el límite de dos horas que se fijaba antiguamente no se justifica en la actualidad; cuando se presenta hemorragia de. be procederse inmediatamente.

Tratamiento de la hemorragia "post_partum".--El éxito de un tratamiento depende de un diagnóstico exacto, y cuando se pre_ senta una hemorragia post-partum es indispensable proceder sin demora a establecer el diagnóstico para proceder en consecuencia. En la práctica es muy común ver que el médico, en su afán de controlar una hemorragia abundante, inicia la aplicación de dro_ gas que en muchas ocasiones no van a producir ningún efecto $\mathrm{y}$, por el contrario, van a demorar un diagnóstico; la rapidez con que se diagnostique la causa de la hemorragia del alumbramien. to es la clave del éxito, y para esto es necesario tener en cuenta los diferentes factores que producen la hemorragia post_partum.

Como ya dijimos, la atonía es el factor principal y por lo tan. to ante una enferma que sangra se debe primero pensar en esta posibilidad, especialmente si el útero no se contrae firmemente. Cuando el parto ha sido espontáneo y se presenta hemorragia, de_ be procederse a una rápida revisión de la cavidad uterina con el fin de hacer la extracción de restos placentarios o coágulos que en estos casos son el factor más común de hemorragia. Si duran_ te el embarazo ha existido una sobredistensión uterina. se deberá pensar en esta causa de inercia e instituir tratamiento con in. fusiones de Pitocín por vía intravenosa lentamente; en los partos laboriosos y prolongados complicados con hemorragia debe insti. tuírse el mismo tratamiento, así como en aquellas eventualidades en las cuales la anestesia profunda o el shock pueden tener algún papel en la hemorragia.

En los partos en los cuales ha habido necesidad de aplicación de fórceps, versiones o extracciones podálicas, se deberá pensar en el origen traumático de la hemorragia, especialmente si el examen muestra una matriz bien contraída, y por lo tanto se de. berá proceder a localizar mediante valvas vaginales el sitio de la hemorragia. Creemos que toda herida del canal genital debe sutu. rarse inmediatamente, bien sea en el cuello, la vagina o la vulva. Estas suturas se deben hacer con catgut simple y aguja traumática; desde luego, cuando se ha producido una ruptura de la ma_ 
triz, debe procederse inmediatamente a la laparatomía para sutu_ rar la brecha o practicar histerectomía, según el caso. No hay que olvidar que en algunos casos pueden presentarse hemorragias de origen traumático en partos espontáneos.

En muchos casos la hemorragia primitivamente es de origen traumático, pero la matriz puede entrar en atonía por retención de coágulos, y el médico trata la atonía uterina infructuosamen. ce, pues tan pronto como se vuelve a llenar la matriz de coágulos, se vuelve a presentar la atonía; lo lógico y racional es hacer una revisión mediante valvas del canal genital.

Hace algunos años se utilizaba el taponamiento intrauterino para aquellos casos de inercia uterina persistente; en la actualidad el pitocín por vía endovenosa en solución dextrosa al 5\% aplica. da lentamente ( 30 gotas por minuto) es, en nuestra opinión, un tratamiento más efectivo y más sencillo que el anterior.

Todas las medidas enumeradas anteriormente para el trata_ miento de la hemorragia producida por los diferentes factores ana. lizados, debe completarse desde luego con el restablecimiento del volumen sanguíneo mediante la transformación, o si ésta no está a la mano mediante plasma o similares. Es de gran utilidad prác_ tica localizar rápidamente una vena superficial que sirva de vía para la transfusión; en los casos de shock intenso es muy prácti_ co la disección de una de las venas del pie y cateterizarla con un tubo de Polietileno. Todos hemos tenido momentos de angustia al fracasar en la localización de una vena para transfusión; la im. portancia de tener la clasificación del grupo sanguíneo y el fac. tor Rh de la enferma no se escapa a nadie.

Sabemos que en algunos casos, especialmente de inercia, fra. casan las medidas enumeradas para control de la hemorragia y se llega un momento en el cual se tiene que plantear la medida extrema de la histerectomía con el fin de salvar una enferma; to. mar esta decisión es una de las grandes responsabilidades del obs. treta y en estos casos es donde se pone a prúeba la serenidad, ex. periencia y pericia del partero; todos sabemos que los textos acon. sejan la histerectomía cuando han fallado las medidas conserva. doras para el control de la hemorragia pero el problema que se plantea al médico es precisamente el de proceder a la operación oportunamente, es decir, cuando el estado de la paciente no sea tan precario y la operación le vaya a servir como medida de sal_ vación.

Es necesario recalcar, en la urgencia y necesidad de establecer un diagnóstico certero, cuando se presenta la hemorragia del alum. 
oramiento; creemos que si el médico tiene la serenidad y el buen juicio de proceder precozmente a buscar la causa de la hemorragia, se evitarán graves complicaciones en la gran mayora de los casos, pero cuando se descontrola, pierde la serenidad y se inician terapéuticas sin tener un diagnóstico, el tiempo perdido mientras se aplican estas medidas puede ser de fatales consecuencias. En muchas ocasiones la simple extracción de un resto placentario o la simple sutura de un desgarro vaginal o cervical hechas oportu_ namente impiden que la paciente entre en shock, el cual en mu. chos casos puede ser fatal.

La hemorragia tardía del post.partum casi siempre se debe a que el utero se encuentra distendido y relajado por restos pla_ centarios retenidos, y en estos casos la paciente debe ser nueva. mente conducida a la sala de partos y se debe proceder a su revi. sión. Cuando la hemorragia va acompañada de distensión uteri. na y dolores intensos que las enfermas describen de mayor in. tensidad a los dolores de parto, es seguro que la causa es una re. tención de coágulos y una simple expresión uterina es el trata. miento ideal.

La hemorragia que continúa anormalmente por los primeros días después del parto, acompañada de subinvolución uterina es casi siempre el resultado de una retención parcial de la placenta, y en estos casos la cavidad uterina debe ser explorada muy sua_ vemente y como el cuello generalmente se está cerrando, la explo. ración debe hacerse con un solo dedo, preferiblemnte a usar la cureta.

La hemorragia post_partum se puede evitar en la gran mayo_ ría de los casos poniendo en práctica las medidas que hemos co_ mentado, pero si se presenta a pesar de estas precauciones es po. sible controlarla mediante los procedimiento adecuados para cada caso y puestos en práctica oportunamente.

Hemorragias por defectos de la coagulación sanguínea. Afibri. nogenemia.-Hasta hace relativamente poco las grandes hemo. rragias post_partum, casi siempre fatales, no tenían una explica. ción satisfactoria. En 1948 se presentó el estudio hecho en el Bos_ ton Lying Hospital, el cual comprendia gran cantidad de pacientes con hemorragias por diferentes causas, a las cuales se les hizo un estudio completo desde el punto de vista de laboratorio y desde entonces se principió a establecer la existencia de severas altera_ ciones de los mecanismos hemostáticos sanguíneos concomitantes con el desprendimiento prematuro de la placenta normalmente insertada; asímismo se estableció que estas alteraciones del po. 
der de coagulación de la sangre eran la consecuencia del despren. dimiento de la placenta y no su causa. Los principales cambios de la coagulación observados fueron la reducción. y en algunas ocasio. nes la desaparición total del fibrinógeno y ocasionalmente un ligero descenso de la concentración de protombina, lo mismo que la apa. rición de una fibrinolisina en la sangre circulante. A estos prime. ros estudios siguieron un sinnúmero de observaciones que vinie. ron a comprobar que la afibrinogenemia era la causa principal de estos estados hemorrágicos; posteriormente se estableció la rela_ ción entre afibrinogenemia y el síndrome de embolía amniótica descrito en 1941 por Steiner y Lushbaugh y más tarde se compro_ bó la existencia del síndrome en los casos de feto muerto y retenido, especialmente en enfermas $\mathrm{Rh}$ negativas isosensibilizadas. Este sindrome se ha descrito también en casos de sepsis graves, quemaduras extensas, cáncer prostático y pulmonar.

Trataremos de hacer una breve reseña de lo que se acepta acerca de los mecanismos de la formación del coágulo. El proceso de la coagulación consiste en esencia en la conversión de una proteina soluble, eỉ fibrinógeno, en una insoluble, la fibrina; esto resulta de la acción de una enzima, la trombina, que parece ser proteolitica. La conversión del fibrinógeno en fibrina es acele. rada por los iones de calcio presentes en la sangre y la sustancia conocida con el nombre genérico de tromboplastina; también se han descrito otros factores en el plasma que aceleran este proceso.

Posiblemente la trombina no existe en cantidades aprecia. bles en la sangre circulante, pero sí existe su predecesor: la pro. combina; la transformación de protombina en trombina ha sido objeto de innumerables controversias, pero se acepta que esta transformación as producida por la tromboplastina, la cual se en cuentra en grandes cantidades en los tejidos animales, incluyen. do la placenta y el líquido amniótico. Parece que el papel de las plaquetas es liberar tromboplastina, iniciando la coagulación. Un gran número de factores pueden contribuir a la actividad de la tromboplastina $\mathrm{y}$ sntre ellos el factor $\mathrm{V}$, o factor lábil, el factor VI, etc.

El plasma humano contiene enzimas proteoliticas capaces de desintegrar el fibrinógeno, la fibrina y varias otras sustancias; estas enzimas se conocen con el nombre de plasminas o fibrino. lisinas, y ordinariamente están en forma inactiva en la circula_ ción (pro_fibrinolisina), pero pueden estar activadas en algunos casos como en el shock y otros estados patológicos, con lo cual se pueden conducir a estados hemorrágicos por este mecanismo. 
El proceso de la coagulación se puede sintetizar así:

Protombina

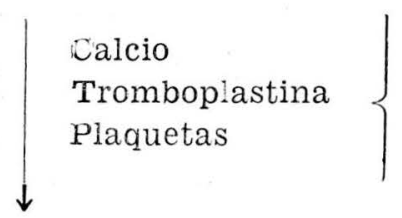

Trombina
Fibrinógeno

Factor antihemof'lico Factor $V$. Ac. Globulina Otros

En la sangre circulanto existe normalmente un equilibrio en. tre las sustancias activadoras de la coagu'ación por una parte y por otra las sustancias inhibidoras o destructoras del coágulo; este delicado balance es sostenido por la normal fluidez de la san. gre y su rápida transformación en coágulos en los sitios donde se altera el endotelio con producción de tromboplastina.

Se sabe que la fibrina puede producirse introduciendo en la sangre circulante tromboplastina o sustancias que la contengan. La tromboplastina puede ser introducida en la circulación por in. yección directa y en la mujer embarazada proviene del tejido pla_ centario.

El tejido placentario humano es muy rico en trombop'astina, y en algunas condiciones patológicas del embarazo grandes canti_ dades pueden pasar a la circulación materna rompiendo el balan. ce existente entre las sustancias procoagulantes y anticoagulantes que sostiene la fluidez de la sangre e iniciando la formación de fibrina mediante la transformación de fibrinógeno; la fibrina así producida se disemina en émbolos y cuando este proceso es muy extenso produce la oclusión circulatoria en órganos tales como el pulmón, riñones, cerebro, etc. Estas oclusiones em. bólicas son microscópicas y de contenido puramente de fibrina, sin contener plasquetas o células, como en el caso del coágulo co_ mún. Paradójicamente en la formación de fibrina se consume gran parte del fibrinógeno circulante, es decir, se produce una afibrinogenemia y como consecuencia una alteración de los mecanis. mos de la coagulación. Cuando el paso de tromboplastina a la circulación sé hace rápidamente en cantidades excesivas, los pe. ligros para la paciente provienen del tromboembolismo, pero si es. te paso se hace lentamente, la lesión predominante será la defi_ brinización de la sangre y la consiguiente falta de coagulación. Es de notar que ambos estados han sido observados en la misma 
paciente; lo mismo se han visto casos en los cuales solamente predomina una de las dos alteraciones.

En la actualidad no existe la menor duda, y se acepta que estos mecanismos explican la etiología de los desórdenes hemorrágicos observados en la embolía amniótica, en el desprendimiento de placenta normalmente insertada y el feto muerto y retenido.

Las razones más aceptadas para explicar el paso de la trom. koplastina a la circulación materna, son las siguientes para ca. da uno de los estados descritos:

En el desprendimiento de la placenta normalmente inserta_ da la tromboplastina pasa mediante el exceso de la tensión que se encuentra en el hematoma retroplacentario. En la embolia am. niótica se explica el paso de tromboplastina por la ruptura de las membranas en su porción alta, dejando así una vía de entrada al líquido para ponerse en contacto con el tejido placentario y los vasos uterinos; también existe otra puerta de entrada, y es la que se encuentra en el cuello cuando se incorpora al segmento a me. dida que progresa el trabajo; como sabemos, esta porción del úte_ ro no está cubierta por las membranas y el líquido viene a quedar en contacto directo con las venas cervicales, lo cual es ayudado a su vez por una contracción uterina exagerada En los casos de feto muerto y retenido el tejido placentario presenta autolisis después de la muerte del feto.

Diagnóstico de laboratorio.-Toda enferma que presenta una de las tres condiciones anotadas anteriormente, es susceptible de complicarse con afibrinogenemia y se debe pensar en esta enti. dad, especialmente si se presentan hemorragias abundantes por vía vaginal y hemorragias por otras vías, tales como encías, heri. das, sitios de punción venosa, sutura de episiotomía, etc.

Se acepta que la cantidad de fibrinógeno normal sin embara_ zo es de más o menos 300 miligramos por 100 c. c. de sangre, y algunos autores sostienen que muy cerca al término el fibrinóge. no llega a cifras de 400 a 440 miligramos por 100 c. c., es decir, 3 gramos de fibrinógeno por litro y más o menos un total de 15 gramos en la sangre circulante. La mayoría de los autores están de acuerdo en aceptar que cuando los niveles de fibrinógeno son inferiores a 100 miligramos por $100 \mathrm{c}$. c. de plasma, se presentan sintomas de hemorragia generalizada es decir, melenas, epis. taxis, equimosis, etc.

Desde el punto de vista de laboratorio, la confirmación de una sospecha de afibrinogenemia es bastante sencilla: se puede ha_ cer la determinación de retracción del coágulo tomando 3 c.c. 
de sangre y colocándolos en un tubo de ensayo y observando si el coágulo se forma en un tiempo normal; cuando no se forma es un indicio bastante sospechoso de que existen niveles críticos de fibrinógeno, es decir, menos de 100 miligramos por 100 c. c.; también se puede determinar el fibrinógeno poniendo 0.10 de c. c. de solución tópica de trombina (1.00:0 U. I. por c. c.) y completando con sangre hasta 1 c. c. Si el coágulo no se forma existe defi. ciencia de fibrinógeno; cuando se forma, pero poco después se desintegra, es porque existen deficiencias pero casi siempre en cantidad superior a 10:0 miligramos por 100 c. c. Las determina_ ciones cuantitativas de fibrinógeno requieren un tiempo no menor de una hora y una técnica de laboratorio bastante dispendiosa.

Las fibrinolisinas pueden determinarse observando el tiempo de lisis del coágulo, es decir, el tiempo que éste gasta para disol. verse completamente; en una persona normal la lisis se presenta después de 2 días de incubación a 37 grados; en algunos estados patológicos es más rápida, pero no tiene valor sino en aquellos estados hemorrágicos en los cuales se presenta en un tiempo no mayor de 2 horas; la determinación de fibrinolisina no es muy importante en los estados de afibrinogenemia, aunque su presen_ cia ha sido descrita parece que no juega un papel esencial.

Afibrinogenemia y desprendimiento de la placenta normal. mente insertada.-No pretendemos hacer un estudio completo del desprendimiento prematuro de la placenta, pero sí debemos ocu. parnos de ella por sus relaciones con el síndrome afibrinogenemia, y por lo tanto analizaremos algunos detalles de esta entidad.

En el año de 1901: De Lee fue quien primero notó la existen. cia de alteraciones "del tipo hemofílico", coexistentes con la apo_ plejía uterina; en 1922 Willson sugirió la existencia de alteracio. nes de la coagulación sanguínea durante el desprendimiento de la placenta; y en 1936 Dieckmann hizo un estudio de la química sanguínea y de la función renal durante el desprendimiento prematuro de la placenta y encontró la disminución del fibrinógeno en la sangre, pero fue Moloney quien primero instituyó el trata_ miento con fibrinógeno como coadyuvante de la transfusión en estos casos.

La afibrinogenemia concomitante con el desprendimiento pre. maturo de la placenta normalmente insertada es producida por el paso de tromboplastina de origen placentario a la circulación materna, gracias al aumento de tensión que existe en el hemato. ma útero_placentario. 
Se sabe que en un 90 a $95 \%$ de los casos el desprendimiento placentario es de grado ligero o moderado, es decir, de uno a dos cotiledones, y en estas circunstancias las manifestaciones clínicas son nulas o muy discretas; es muy común que el diagnóstico se haga retrospectivamente al hacer el examen de la placenta. En este grupo de pacientes la mortalidad materna es nula y los sín. tomas consisten en dolor localizado en el sitio del desprendimien. to, hemorragia vaginal discreta, sufrimiento fetal y excepcional. mente estado pasajero de shock.

En un 5 a $10 \%$ de los casos el desprendimiento es extenso, más de la tercera parte del órgano, y los síntomas consisten en estado de shock profundo, aumento del tamaño de la matriz, aus. cultación fetal negativa y dolor abdominal intenso; es el cuadro conocido clásicamente con el nombre de apoplejía uterina o úte. ro de Couvelaire, en el cual existe disociación de la fibra muscular uterina, infiltración sanguínea de los parametrios y disocia_ ción del peritóneo.

La apreciación del grado y severidad del desprendimiento es un factor definitivo en el buen éxito del tratamiento y hoy día se puede obtener una idea bastante clara de esta intensidad median. te la apreciación de los sintomas y signos clínicos y así como los datos suministrados por el laboratorio.

La relación entre la toxemia del embarazo y desprendimiento de la placenta normalmente insertada es un hecho aceptado, pe. ro la intensidad del desprendimiento no es índice del grado de toxemia; un 50 a $70 \%$ de las pacientes con abruptio-placentae no presentan hipertensión ni albuminuria y la presencia de esta úl. tima no siempre es índice de toxemia, ya que puede ser produci. da por el hematoma retenido; por otra parte es excepcional la afibrinogenemia como complicación de la eclampsia.

Conducta.-A medida que se han ido conociendo mejor los síndromes de afibrinogenemia, shock y desprendimiento prema_ turo de la placenta, la conducta se ha ido sistematizando en la mayoría de los centros hospitalarios bien dotados, pero desde lue. go siguen existiendo diferentes escuelas y diferentes maneras de afrontar el problema.

En los casos de desprendimiento moderado no existe discre. pancia en cuanto a tratamiento; el primer paso debe ser la ruptu. ra de las membranas, seguida de un tiempo prudencial de espera durante el cual deben presentarse las contracciones del parto y si esto no se sucede podrá recurrir al pitocín endovenoso bajo control, pero si después de 12 horas no se presenta el trabajo, debe 
pensarse en la cesárea, y ésta tendrá su indicación si se presenta sufrimiento fetal o si la hemorragia vaginal aumenta o se pre. sentan otros sintomas que indiquen aumento en el desprendimien. to; con este plan de tratamiento la cesárea es la excepción y la mortalidad fetal es un $10 \%$ e imputable especialmente a la pre. maturez, membrana hialina, etc.

En las formas severas de desprendimiento el tratamiento se dirige especialmente a salvar a la madre ya que la mortalidad fe. tal es de un 100 por 100. La práctica de la cesárea hecha inmedia_ tamente en una enferma que se encuentra en intenso shock es fatal, y hoy día es más aconsejable combatir previamente este estado y constatar la existencia de afibrinogenemia. El tratamien. to se puede esquematizar así: a) Tratamiento de shock por los medios usuales. b) Ruptura de las membranas. c) Tomas de san. gre para observar la retracción del coágulo hechas cada hora, te. niendo en cuenta que en muchos casos el coágulo se forma, pero al cabo de pocos minutos se fragmenta y se disuelve. Si existe afi. brinogenemia se debe iniciar el tratamiento con esta sustancia. Es lo común observar que la enferma salga de su estado de shock e inicie el trabajo de parto, el cual se puede dejar evolucionar siempre y cuando que no se prevea la menor dificultad, pues si esto sucede es aconsejable la cesárea, ya que si el parto se hace laborioso por cualquier circunstancia, la enferma puede presentar un nuevo estado de shock de caracteres irreversibles.

En muchos centros hospitalarios la cesárea es el tratamien. to de elección, pero hoy día con los conocimientos sobre la afibri_ nogenemia se ha observado que esta entidad se presenta con menos frecuencia después de los partos por vía vaginal y por otra parte el concepto según el cual el útero de Couvelaire es incapaz de contraerse y la hemorragia por atonía uterina es fatal, ha si_ do modificado; la experiencia ha demostrado que úteros seria. mente' afectados son capaces de contraerse, producir parto espon. táneo sin la menor hemorragia por atonía.

En los centros médicos bien dotados la histerectomía ha ce. dido su paso al tratamiento médico, pues es innegable que con su práctica solamente se agregan más fuentes de hemorragia cuan. do existe afibrinogenemia. El argumento de que los estados he morrágicos se acentúan mientras el feto esté dentro de la cavi. dad uterina y que la práctica de la cesárea e histerectomía elimi. nan este factor, ha sido rectificado con la comprobación de que la ruptura de las membranas detienen el proceso. 
En la segunda Convención de Ginecología y Obstetricia reu. nida en Cali, la Delegación antioqueña presentó varias observa. ciones de afibrinogenemia tratadas con éxito con A. C. T. H. La literatura médica que hemos tenido oportunidad de consultar es bastante pesimista sobre el tratamiento con esta sustancia, y las pocas observaciones que conocemos de algunos colegas tam. bién demuestran un fracaso completo con esta droga.

Embolía amniótica-Es ésta una causa importante de hemorragia generalizada durante el parto; este sindrome fue descrito inicialmente en 1941 por Steiner y Lushbaug y tiene como causa la entrada al torrente circulatorio inmediatamente antes del parto, durante éste o después de células amnióticas, lanugo, etc. En la mayoría de las observaciones se destaca el hecho de que los partos han sido demasiado rápidos. Desde el punto de vista clínico se describe una forma fulminante en la cual la paciente entra en severa insuficiencia respiratoria, con disnea, cianosis, shock y muerte en pocos minutos; en otros casos el shock se presenta gra. dualmente, los síntomas respiratorios no son tan acentuados y son estos casos en los cuales existe la complicación de la afibrinoge. nemia. La autopsia practicada a las pacientes que mueren por es. ta causa muestra embolización por líquido amniótico con células epiteliales del amnios, lanugo, etc., en los vasos pulmonares.

La afibrinogenemia ha podido ser demostrada experimental_ mente en perros mediante la inyección de líquido amniótico.

Como ya dijimos se acepta como causa de defibrinización el paso de tromboplastina de origen placentario a la circulación ma_ terna en el curso de un trabajo intenso, bien sea por las venas cer. vicales expuestas al contacto del líquido o bien sea por rupturas altas de las membranas que van a permitir su disociación y por lo tanto entrar en contacto con el tejido placentario.

Toda paciente que sobreviva al accidente inicial deberá ser sometida a los tratamientos rutinarios del shock y si se comprue. ba la afibrinogenemia se procederá a las inyecciones de fibrinógeno hasta obtener concentraciones de unos 150 miligramos por 100 c. c. niveles éstos que parecen ser suficientes para provocar la formación de un coágulo aceptable.

Es importante anotar que en algunos casos la hemorragia no ha cedido, a pesar de haberse corregido la afibrinogenemia y pa. rece que en éstos se ha presentado una severa trombocitopenia muy posiblemente como resultado de las transfusiones masivas.

Feto muerto y retenido.-En el curso de las investigaciones de los mecanismos de coagulación durante los estados anterior. 
mente descritos se observaron pacientes con fetos muertos y rete. nidos que sangraban copiosamente después del parto y en las cua_ les se comprobó la afibrinogenemia. En 1950 Weiner, Aeid y Robb presentaron casos comprobados del síndrome afibrinogenemia complicando la situación de feto muerto y retenido, pero en 1911 De Lee había descrito casos similares sin poder dar una explica. ción satisfactoria a la hemorragia. El porcentaje de afibrinogene. mia en casos de feto muerto y retenido es relativamente más al_ to en relación con los otros estados en que se observa; parece que hasta el presente no ha sido descrita antes del cuarto mes de em. barazo, así como tampoco antes de cinco semanas de estar el feto muerto y retenido; la asociación con eritroblastosis es muy co. mún, pero no siempre existe isoinmunización en las enfermas que la presentan. Se han descrito casos de afibrinogenemia varios días después de haberse expulsado un feto muerto y retenido.

Es importante anotar que en la mayoría de los casos se pue. de hacer un diagnóstico antes de presentarse el parto, especial_ mente cuando se observan tendencias hemorrágicas, bien sea por via nasal, gingival, etc.

Es aconsejable ordenar determinaciones de fibrinógeno antes de proceder a provocar el parto y si se encuentra deficiencia de esta sustancia lo ideal es tratar la enferma previamente, es de. cir. hacer un tratamiento profiláctico. Es también importante advertir a la enferma que reporte' a su médico si observa cual. quier tendencia a la hemorragia.

Como ya dijimos anteriormente, se acepta hoy día que las tromboplastinas desfibrinizantes provienen de autolisis de la de. cidua o del tejido placentario y el paso de estas sustancias es fa_ vorecido por las contracciones tipo Braxton_Hicks o por las con_ tracciones del parto.

La afibrinogenemia adquirida es indudablemente una compli. cación potencial para cualquier enferma que presente los tres es_ tados descritos y por lo tanto el obstetra deberá tener siempre en mente su posible existencia, así como también será forzoso en el futuro que en las salas de parto existan los elementos de labora. torio necesarios para hacer una rápida determinación de fibrinógeno, al mismo tiempo que debemos aspirar a tener este elemento a la mano, ya que sin él el tratamiento de esta complicación no pasa de ser empírico y teórico. 


\section{BIBLIOGRAFIA}

WILLSON J. ROBERT.-Prevention and treatment of post_partum hemorrhage. The Surgical Clinics of North America. 34-1591. 1954.

L. NEWBLIN F. PAXSON.-The Management of Afibrinogenemia. The Surgical Clinics of North América. 34. 1601. 1954.

ALEXANDER BENJAMIN.-Coagulation Hemorrhage and Thrombosis New Eng. J. Med. 252-526. 1955.

RATNOFF OSCAR D., PRITCHARD JACK A., COLOPY JOANE.-Hemo_ rrhage States during pregnancy. New Eng. J. Med. 253. 63 y 97. 1955.

REID E. DUNCANN, WEINER E. ALBERT, ROBY C. CHARLES.-Intravascular clotting and Afibrinogenemia. Presumptive Lethal Factor in the Syn. drome of Amniotic Fluid Emobolism. Am. J. Obst. \& Gynec. 66. 465. 1953.

REID E. DUNCANN, WEINER E. ALBERT, ROBY C. CHARLES.-Incoagulable blood in severe premature separation of the placenta. A method of mangement. Am. J. Obst. \& Gynec. 66. 475. 1953.

REID E. DUNCAN, WEINER E. ALBERT, ROBY C. CHARLES, DIA. MONDLOUIS K.-Maternal afibrinogenemia associated with long standing in. trauterine fetal death. Am. J. Obst. \& Gynec. 66. 500. 1953.

DUGALD BAIR.-Combines Text Book of Obstetric and Ginecology. Fifth Edition. L \& S. Livingstone Ltd.

SHULMAN ABRAHAM, RATZAN WILFORD J., PATERSON N. J., GROS_ BARD PAUL, PASSAIC N. J., LAWRENCE ARTHUR C., PATTERSON N. J. Post-partum Hemorrhage. Etiology \& Management. Am. J. Obst. \& Gynec. 71. 37. 1956.

WEEKES LEROY R. O'TOOLE DANIEL M.-Post-partum Hemorrhage. Am. Obst. \& Gynec. 71. 45. 1956.

KLEIN MICHAEL, BISKIND JOHN I., SILVERBERG ALBERT.-Post. partum Hemorrhage Due to Depletion of Fibrinogen from the Circulating Blood Stream. Am. Obst. \& Gynec. 71. 51. 1956. 